\title{
A complete classification of doubly even self-dual codes of length $40^{*}$
}

\author{
Koichi Betsumiya \\ Graduate School of Science and Technology \\ Hirosaki University \\ Hirosaki 036-8561, Japan \\ betsumi@cc.hirosaki-u.ac.jp \\ Masaaki Harada \\ Department of Mathematical Sciences \\ Yamagata University \\ Yamagata 990-8560, Japan, and \\ PRESTO, Japan Science and Technology Agency (JST) \\ Saitama 332-0012, Japan \\ mharada@sci.kj.yamagata-u.ac.jp \\ Akihiro Munemasa \\ Graduate School of Information Sciences \\ Tohoku University \\ Sendai 980-8579, Japan \\ munemasa@math.is.tohoku.ac.jp
}

Submitted: June 3, 2011; Accepted: July 27, 2012; Published: Aug 9, 2012

Mathematics Subject Classifications: 94B05

\begin{abstract}
A complete classification of binary doubly even self-dual codes of length 40 is given. As a consequence, a classification of binary extremal self-dual codes of length 38 is also given.
\end{abstract}

Keywords: self-dual code; weight enumerator; mass formula

*This work was supported by JST PRESTO program. 


\section{Introduction}

As described in [26], self-dual codes are an important class of linear codes for both theoretical and practical reasons. It is a fundamental problem to classify self-dual codes of modest lengths and much work has been done towards classifying self-dual codes over $\mathbb{F}_{q}$ for $q=2$ and 3 , where $\mathbb{F}_{q}$ denotes the finite field of order $q$ and $q$ is a prime power (see [26]).

Codes over $\mathbb{F}_{2}$ are called binary and all codes in this paper are binary. The dual code $C^{\perp}$ of a code $C$ of length $n$ is defined as $C^{\perp}=\left\{x \in \mathbb{F}_{2}^{n} \mid x \cdot y=0\right.$ for all $\left.y \in C\right\}$, where $x \cdot y$ is the standard inner product. A code $C$ is called self-dual if $C=C^{\perp}$. A self-dual code $C$ is doubly even if all codewords of $C$ have weight divisible by four, and singly even if there is at least one codeword of weight $\equiv 2(\bmod 4)$. It is known that a self-dual code of length $n$ exists if and only if $n$ is even, and a doubly even self-dual code of length $n$ exists if and only if $n$ is divisible by eight. The minimum weight $d$ of a self-dual code of length $n$ is bounded by $d \leqslant 4\left\lfloor\frac{n}{24}\right\rfloor+6$ if $n \equiv 22(\bmod 24), d \leqslant 4\left\lfloor\frac{n}{24}\right\rfloor+4$ otherwise [20] and [25]. A self-dual code meeting the bound is called extremal.

Two codes $C$ and $C^{\prime}$ are equivalent, denoted $C \cong C^{\prime}$, if one can be obtained from the other by permuting the coordinates. An automorphism of $C$ is a permutation of the coordinates of $C$ which preserves $C$. The set consisting of all automorphisms of $C$ is called the automorphism group of $C$ and it is denoted by $\operatorname{Aut}(C)$.

A classification of doubly even self-dual codes was done for lengths 8,16 in [23], for length 24 in [24] and for length 32 in [9]. For length 40, only some partial classifications have been done by various authors. Extremal doubly even self-dual codes of length 40 with automorphism of a prime order $p$ having $c$ cycles have been classified for $(p, c)=$ $(19,2),(7,5),(5,4)$ in $[28],(p, c)=(3,6)$ in $[6],(p, c)=(3,8)$ in $[16]$, and $(p, c)=(5,8)$ in [29]. The main aim of this paper is to give a classification of doubly even self-dual codes of length 40.

Theorem 1. There are 94343 inequivalent doubly even self-dual codes of length 40, 16470 of which are extremal.

As a summary, we list in Table 1 the total number $N_{T}(n)$ of inequivalent doubly even self-dual codes of length $n$ and the number $N_{d}(n)$ of inequivalent doubly even self-dual codes of length $n(n=8,16, \ldots, 40)$ and minimum weight $d(d=4,8)$.

Table 1: Number of doubly even self-dual codes

\begin{tabular}{c|c|c|c}
\hline Length $n$ & $N_{T}(n)$ & $N_{4}(n)$ & $N_{8}(n)$ \\
\hline 8 & 1 & 1 & - \\
16 & 2 & 2 & - \\
24 & 9 & 8 & 1 \\
32 & 85 & 80 & 5 \\
40 & 94343 & 77873 & 16470 \\
\hline
\end{tabular}


A classification of singly even self-dual codes of lengths up to 36 is known [3], [4], [9], [11], [23], [24]. As a consequence of Theorem 1, we give a classification of extremal singly even self-dual codes of length 38 .

Generator matrices of all inequivalent doubly even self-dual codes of length 40 and extremal self-dual codes of length 38 can be obtained electronically from [12]. All computer calculations in this paper were done by MAGMA [5].

\section{Classification method}

In this section, we describe how to complete a classification of doubly even self-dual codes of length 40 .

\subsection{Preliminaries}

The weight enumerator of a doubly even self-dual code of length 40 can be written as:

$$
\begin{aligned}
1+A_{4} y^{4}+\left(285+24 A_{4}\right) & y^{8}+\left(21280+92 A_{4}\right) y^{12} \\
& +\left(239970-600 A_{4}\right) y^{16}+\left(525504+966 A_{4}\right) y^{20}+\cdots+y^{40},
\end{aligned}
$$

where $A_{w}$ denotes the number of codewords of weight $w$ (see e.g. [20]).

The number of distinct doubly even self-dual codes of length $n$ is given [19] by the formula:

$$
\prod_{i=0}^{n / 2-2}\left(2^{i}+1\right)
$$

King [18] determined the number of distinct extremal doubly even self-dual codes of length 40. Let $N(40, d)$ denote the number of distinct doubly even self-dual codes of length 40 and minimum weight $d(d=4,8)$. Then we have

$$
\begin{aligned}
& N(40,4)=4009357722800739726876619952910304312989584368968750, \\
& N(40,8)=10263335567003567415076803513287627980544163840000000 .
\end{aligned}
$$

\subsection{Minimum weight 4}

Let $C$ be a singly even self-dual code and let $C_{0}$ denote the subcode of codewords having weight $\equiv 0(\bmod 4)$. Then $C_{0}$ is a subcode of codimension 1 . The shadow $S$ of $C$ is defined to be $C_{0}^{\perp} \backslash C$ [10]. There are cosets $C_{1}, C_{2}, C_{3}$ of $C_{0}$ such that $C_{0}^{\perp}=C_{0} \cup C_{1} \cup C_{2} \cup C_{3}$, where $C=C_{0} \cup C_{2}$ and $S=C_{1} \cup C_{3}$.

Proposition 2 (Brualdi and Pless [8]). Let $C$ be a self-dual code of length $n \equiv 6(\bmod 8)$. Let $C_{0}, C_{1}, C_{2}$ and $C_{3}$ be as above. Then

$$
\begin{aligned}
& C^{*}=\left\{(v, 0,0) \mid v \in C_{0}\right\} \cup\left\{(v, 1,1) \mid v \in C_{2}\right\} \\
& \cup\left\{(v, 1,0) \mid v \in C_{1}\right\} \cup\left\{(v, 0,1) \mid v \in C_{3}\right\}
\end{aligned}
$$

is a doubly even self-dual code of length $n+2$. 
There are 519492 inequivalent self-dual codes of length 36 [11]. By considering the direct sum of the unique self-dual code of length 2 and each of these codes, we have 519492 self-dual codes of length 38 and minimum weight 2. By Proposition 2, 519492 doubly even self-dual codes of length 40 and minimum weight 4 are constructed.

We examine the equivalence or inequivalence of codes as follows. Let $C$ be a doubly even self-dual code of length 40 and minimum weight $d(d=4,8)$. Let $M(C)$ be the $A_{8} \times 40$ matrix with rows composed of the codewords of weight 8 in $C$, where the $(1,0)$-matrix $M(C)$ is regarded as a matrix over $\mathbb{Z}$. We define

$$
N(C)= \begin{cases}\left\{n_{i j} \mid 1 \leqslant i, j \leqslant 40\right\} \backslash\{57\} & \text { if } C \text { is extremal } \\ \left\{n_{i j} \mid 1 \leqslant i, j \leqslant 40\right\} & \text { otherwise }\end{cases}
$$

where $n_{i j}$ is the $(i, j)$-entry of $M(C)^{T} M(C)$, and $M(C)^{T}$ denotes the transposed matrix of $M(C)$. The codewords of weight $w$ in $C$ are calculated by the Magma function Words. Note that the codewords of weight 8 in $C$ form a $1-(40,8,57)$ design when $C$ is extremal. This means that $n_{i i}=57$ for any $i(i=1,2, \ldots, 40)$ and $\max \left\{n_{i j} \mid 1 \leqslant i, j \leqslant 40\right\}=57$ when $C$ is extremal. Then we consider the following:

$$
\alpha(C)=\left(\# \operatorname{Aut}(C), A_{4}, \max N(C), \min N(C), \# N(C)\right) .
$$

The automorphism group $\operatorname{Aut}(C)$ of the code $C$ is calculated by the MAGMA function AutomorphismGroup. Of course, $C$ and $C^{\prime}$ are inequivalent if $\alpha(C) \neq \alpha\left(C^{\prime}\right)$. For a given set of codes, we divided into classes where each class contains codes $C$ with identical $\alpha(C)$. Then we divided the codes in each class into equivalence classes. This was done by the MAGMA function IsIsomorphic.

In this way, we checked equivalences among the above 519492 doubly even self-dual codes of length 40 and minimum weight 4 . Then we obtained the set $\mathcal{C}_{40,4}$ of 77873 inequivalent doubly even self-dual codes with minimum weight 4 satisfying

$$
\sum_{C \in \mathcal{C}_{40,4}} \frac{40 !}{\# \operatorname{Aut}(C)}=N(40,4) .
$$

This shows that there is no other doubly even self-dual code of length 40 and minimum weight 4 . The numbers $N\left(A_{4}\right)$ of doubly even self-dual codes of length 40 containing $A_{4}$ codewords of weight 4 are listed in Table 2.

\subsection{Minimum weight 8}

For a set of coordinates $I \subset\{1,2, \ldots, n\}$, let $\pi: \mathbb{F}_{2}^{n} \rightarrow \mathbb{F}_{2}^{t}, \pi^{\prime}: \mathbb{F}_{2}^{n} \rightarrow \mathbb{F}_{2}^{n-t}$ be the projection to the set of coordinates $I, I^{\prime}$, respectively, where $I^{\prime}=\{1, \ldots, n\} \backslash I$ and $\# I=t$. For a code $C$ of length $n$, the punctured code and the shortened code of $C$ on the set of coordinates $I$ are the codes $\pi^{\prime}(C)$ and $\left\{\pi^{\prime}(c) \mid c \in C, \pi(c)=\mathbf{0}\right\}$, respectively, where $\mathbf{0}$ denotes the zero vector.

If $C$ is a doubly even code containing the all-one vector 1 , then we denote by $q_{C}$ : $C^{\perp} / C \rightarrow \mathbb{F}_{2}$ the map defined by $q_{C}(x+C)=\frac{\operatorname{wt}(x)}{2} \bmod 2$, where $\operatorname{wt}(x)$ denotes the weight of $x$. It is easy to verify that the map $q_{C}$ is well-defined. 
Table 2: Number of doubly even self-dual codes of length 40

\begin{tabular}{c|c|c|c|c}
\hline \multicolumn{5}{c}{$\left(A_{4}, N\left(A_{4}\right)\right)$} \\
\hline$(0,16470)$ & $(13,382)$ & $(26,47)$ & $(40,12)$ & $(64,3)$ \\
$(1,20034)$ & $(14,374)$ & $(27,16)$ & $(41,1)$ & $(66,1)$ \\
$(2,17276)$ & $(15,231)$ & $(28,38)$ & $(42,9)$ & $(70,3)$ \\
$(3,12168)$ & $(16,236)$ & $(29,13)$ & $(43,3)$ & $(72,1)$ \\
$(4,8471)$ & $(17,143)$ & $(30,29)$ & $(44,7)$ & $(74,1)$ \\
$(5,5552)$ & $(18,160)$ & $(31,7)$ & $(46,7)$ & $(78,1)$ \\
$(6,3916)$ & $(19,100)$ & $(32,22)$ & $(48,4)$ & $(90,1)$ \\
$(7,2610)$ & $(20,104)$ & $(33,3)$ & $(50,4)$ & $(92,1)$ \\
$(8,1932)$ & $(21,54)$ & $(34,25)$ & $(52,6)$ & $(94,2)$ \\
$(9,1243)$ & $(22,90)$ & $(35,3)$ & $(54,2)$ & $(106,1)$ \\
$(10,1093)$ & $(23,37)$ & $(36,11)$ & $(56,1)$ & $(134,1)$ \\
$(11,669)$ & $(24,59)$ & $(37,4)$ & $(58,4)$ & $(190,1)$ \\
$(12,605)$ & $(25,26)$ & $(38,11)$ & $(62,2)$ & \\
\hline
\end{tabular}

Let $C_{i}$ be a doubly even code of length $n_{i}$ containing $\mathbf{1}$, for $i=1,2$. A bijective linear map

$$
f: C_{1}^{\perp} / C_{1} \rightarrow C_{2}^{\perp} / C_{2}
$$

is called an isometry if $q_{C_{1}}=q_{C_{2}} \circ f$. The set of isometries (4) is denoted by $\Phi\left(C_{1}, C_{2}\right)$. Note that an isometry exists only if $n_{1}-n_{2}=2\left(\operatorname{dim} C_{1}-\operatorname{dim} C_{2}\right)$ and $n_{1} \equiv n_{2}(\bmod 8)$. For an isometry $f \in \Phi\left(C_{1}, C_{2}\right)$, we define a code

$$
D\left(C_{1}, C_{2}, f\right)=\left\{\left(x_{1}, x_{2}\right) \mid x_{1} \in C_{1}^{\perp}, x_{2} \in f\left(x_{1}+C_{1}\right)\right\} \subset \mathbb{F}_{2}^{n_{1}+n_{2}},
$$

It is easy to see that $D\left(C_{1}, C_{2}, f\right)$ is a doubly even self-dual code. Conversely, every doubly even self-dual code of length $n_{1}+n_{2}$ containing a codeword of weight $n_{1}$ can be constructed by this method. Indeed, let $x$ be a codeword of weight $n_{1}$ in a doubly even self-dual code $C$ of length $n_{1}+n_{2}$. Let $C_{2}$ (resp. $C_{1}$ ) be the shortened code of $C$ on the support (resp. the complement of the support) of $x$. Then $C_{1}$ and $C_{2}$ are doubly even codes. Moreover, $C_{2}^{\perp}$ (resp. $C_{1}^{\perp}$ ) is the punctured code of $C$ on the support (resp. the complement of the support) of $x$. Let $\pi$ and $\pi^{\prime}$ denote the projections onto the support of $x$ and the complement of the support of $x$, respectively. We define $f: C_{1}^{\perp} / C_{1} \rightarrow C_{2}^{\perp} / C_{2}$ by $f\left(x_{1}+C_{1}\right)=\pi^{\prime}(x)+C_{2}$, where $x$ is a codeword of $C$ satisfying $\pi(x)=x_{1}$. Then $D\left(C_{1}, C_{2}, f\right)$ is equivalent to $C$.

For fixed codes $C_{1}, C_{2}$, the resulting code $D\left(C_{1}, C_{2}, f\right)$ depends on the choice of an isometry $f$. However, some of these codes are equivalent to each other. We will give a sufficient condition for two resulting codes to be equivalent. We need this criterion to reduce the amount of calculation to be reasonable.

First, we define some groups. For a doubly even code $C$ containing $\mathbf{1}$, we denote by $\mathcal{G}_{0}(C)$ the subgroup of $\operatorname{GL}\left(C^{\perp} / C\right)$ induced by the action of $\operatorname{Aut}(C)$ on the linear space $C^{\perp} / C$ and denote by $\mathcal{G}_{1}(C)$ the subgroup $\Phi(C, C)$ of $\mathrm{GL}\left(C^{\perp} / C\right)$. By the definition, the 
group $\mathcal{G}_{0}(C)$ is a subgroup of $\mathcal{G}_{1}(C)$. If we replace $f$ by $\sigma_{2} \circ f \circ \sigma_{1}$, where $\sigma_{i} \in \mathcal{G}_{0}\left(C_{i}\right)$, then the resulting codes are equivalent, that is,

$$
D\left(C_{1}, C_{2}, f\right) \cong D\left(C_{1}, C_{2}, \sigma_{2} \circ f \circ \sigma_{1}\right)
$$

This means that, in order to enumerate the set of codes $\left\{D\left(C_{1}, C_{2}, h\right) \mid h \in \Phi\left(C_{1}, C_{2}\right)\right\}$ up to equivalence, we first fix $f \in \Phi\left(C_{1}, C_{2}\right)$, and it suffices to enumerate the codes $D\left(C_{1}, C_{2}, f \circ g\right)$, where $g$ runs through a set of representatives for the double cosets

$$
\left(f^{-1} \circ \mathcal{G}_{0}\left(C_{2}\right) \circ f\right) \backslash \mathcal{G}_{1}\left(C_{1}\right) / \mathcal{G}_{0}\left(C_{1}\right) .
$$

We now apply this method with $\left(n_{1}, n_{2}\right)=(16,24)$ in order to classify extremal doubly even self-dual codes of length 40 . Note that from the weight enumerator (1), such a code has a codeword of weight 16 . This means that every extremal doubly even self-dual code of length 40 is equivalent to $D\left(C_{1}, C_{2}, f\right)$ for some doubly even code $C_{1}$ of length 16 containing $\mathbf{1}$, some doubly even code $C_{2}$ of length 24 containing $\mathbf{1}$, and $f \in \Phi\left(C_{1}, C_{2}\right)$. All doubly even codes of lengths 16 and 24 can be found in [21].

However, if $\operatorname{dim} C_{1} \leqslant 2$, then the degree of $\mathcal{G}_{1}\left(C_{1}\right) \subset \mathrm{GL}\left(C_{1}^{\perp} / C_{1}\right)$ as a permutation group is too large to perform the double coset enumeration, so we only enumerated codes $D\left(C_{1}, C_{2}, f\right)$, where $C_{1}$ is a doubly even code of length 16 with $\operatorname{dim} C_{1} \geqslant 3$. Here, the group $\mathcal{G}_{1}\left(C_{1}\right)$ was constructed by the MAGMA function GOPlus, and the double coset enumeration was performed using the MAGMA function DoubleCosetRepresentatives. Then we classified the resulting codes using the method described in the previous subsection. In this way, we obtained a set of pairwise inequivalent 16468 extremal doubly even self-dual codes of length 40. It turns out that there are two other codes. One is the code with automorphism group of order 6840 constructed in [28]. The other is the code $H^{(1234)} B_{6}^{\prime}$ in the notation of [29] and this code has automorphism group of order 120 .

In this way, we obtained the set $\mathcal{C}_{40,8}$ of 16470 inequivalent extremal doubly even self-dual codes satisfying

$$
\sum_{C \in \mathcal{C}_{40,8}} \frac{40 !}{\# \operatorname{Aut}(C)}=N(40,8) .
$$

From (3) and (6), it follows that there is no other doubly even self-dual code of length 40. This explains the number $N(0)$ of extremal doubly even self-dual codes of length 40 listed in Table 2. Therefore, we have Theorem 1.

\section{Some properties}

In this section, we give some properties of doubly even self-dual codes of length 40 .

The covering radius of a code $C$ of length $n$ is the smallest integer $R$ such that spheres of radius $R$ around codewords of $C$ cover the space $\mathbb{F}_{2}^{n}$. It is known that the covering radius is the same as the largest value among weights of cosets. Here, the weight of a coset is the smallest weight of a vector in the coset. The covering radius is a basic and 
important geometric parameter of a code. Assmus and Pless [2] began the study of the covering radii of (extremal) doubly even self-dual codes.

Let $R_{40, d}$ be the covering radius of a doubly even self-dual code of length 40 and minimum weight $d(d=4,8)$. Then, by the sphere-covering bound and the Delsarte bound (see [2]), $6 \leqslant R_{40,8} \leqslant 8$ and $6 \leqslant R_{40,4} \leqslant 10$. In Table 3 , we list the numbers $N(d, R)$ of doubly even self-dual codes with minimum weight $d$ and covering radius $R$. This was calculated by the MAgma function CoveringRadius. From the above calculation, we have the following:

Proposition 3. There is no doubly even self-dual code of length 40 with covering radius 6.

Remark 4. In [14], based on a preprint by Michio Ozeki, the non-existence of an extremal doubly even self-dual code with covering radius 6 was announced. However, unfortunately, his preprint contained an error and, in his paper [22] he withdrew the above announcement. From the above calculation, the non-existence of an extremal doubly even self-dual code with covering radius 6 was verified.

Remark 5. The two extremal doubly even self-dual codes with covering radius 7 can be found in [13] and [14].

Table 3: Covering radii of doubly even self-dual codes

\begin{tabular}{c|c|c}
\hline$R$ & $N(4, R)$ & $N(8, R)$ \\
\hline 6 & 0 & 0 \\
7 & 23 & 2 \\
8 & 76768 & 16468 \\
9 & 954 & - \\
10 & 128 & - \\
\hline
\end{tabular}

Now we give some properties of extremal doubly even self-dual codes of length 40 . Let $\sigma$ be an automorphism of odd prime order $p$. If $\sigma$ has $c$ independent $p$-cycles and $f$ fixed points, then $\sigma$ is said to be of type $p$ - $(c, f)$. All extremal doubly even self-dual codes of length 40 with automorphism of type $p$ - $(c, f)$ are known for $p \geqslant 5$ (see [15, Table 3]). The cases with $(p, c)=(3,6)$ and $(3,8)$ were considered in [6] and [16], respectively. The numbers $N(p, c)$ of inequivalent extremal doubly even self-dual codes with automorphism of type $p-(c, f)$ are listed in Table 4 for $(p, c)=(3,6),(3,10)$ and $(3,12)$. It is claimed in $[6$, Theorem 12] that $N(3,6)=16$. However, we verified that $N(3,6)=17$. Since the list of the 16 codes is not available, we are unable to compare the result with ours.

Proposition 6. There are 17, 70 and 322 inequivalent extremal doubly even self-dual codes of length 40 with automorphism of types $3-(6,22), 3-(10,10)$ and $3-(12,4)$, respectively. 
Table 4: $N(p, c)$ for $(p, c)=(3,6),(3,10)$ and $(3,12)$

\begin{tabular}{c|c|c|c}
\hline$(p, c)$ & $(3,6)$ & $(3,10)$ & $(3,12)$ \\
\hline$N(p, c)$ & 17 & 70 & 322 \\
\hline
\end{tabular}

Table 5: Orders of automorphism groups

\begin{tabular}{c|c|c|c|c}
\hline \multicolumn{5}{c}{$(\#$ Aut, $N(\#$ Aut $)$} \\
\hline$(1,10400)$ & $(36,1)$ & $(256,21)$ & $(3072,3)$ & $(61440,1)$ \\
$(2,3538)$ & $(38,1)$ & $(288,4)$ & $(3840,1)$ & $(65536,1)$ \\
$(3,43)$ & $(40,5)$ & $(320,1)$ & $(4096,1)$ & $(110592,1)$ \\
$(4,1189)$ & $(48,34)$ & $(384,12)$ & $(4608,2)$ & $(147456,1)$ \\
$(5,2)$ & $(60,2)$ & $(512,16)$ & $(5376,1)$ & $(245760,1)$ \\
$(6,68)$ & $(64,75)$ & $(576,3)$ & $(6144,7)$ & $(737280,1)$ \\
$(8,459)$ & $(72,4)$ & $(720,2)$ & $(6840,1)$ & $(786432,1)$ \\
$(10,8)$ & $(96,12)$ & $(768,7)$ & $(9216,1)$ & $(983040,1)$ \\
$(12,80)$ & $(114,1)$ & $(1024,3)$ & $(12288,2)$ & $(1474560,1)$ \\
$(16,233)$ & $(120,5)$ & $(1296,1)$ & $(16384,1)$ & $(5505024,1)$ \\
$(18,1)$ & $(128,46)$ & $(1536,10)$ & $(18432,1)$ & $(8257536,1)$ \\
$(20,4)$ & $(144,4)$ & $(1728,1)$ & $(20480,1)$ & $(44236800,1)$ \\
$(24,41)$ & $(160,1)$ & $(1920,1)$ & $(20736,1)$ & $(82575360,1)$ \\
$(30,2)$ & $(192,12)$ & $(2048,4)$ & $(32768,1)$ & \\
$(32,70)$ & $(240,2)$ & $(2688,1)$ & $(49152,3)$ & \\
\hline
\end{tabular}

In Table 5, we list the numbers $N$ (\# Aut) of extremal doubly even self-dual codes with automorphism groups of order \# Aut.

As we mentioned at the end of Subsection 2.3, we have the following:

Proposition 7. Let $C_{x}$ denote the shortened code of $C$ on the complement of the support of a codeword $x$. Then there are two inequivalent extremal doubly even self-dual codes $C$ of length 40 such that $\operatorname{dim} C_{x} \leqslant 2$ for all $x \in C$ with $\operatorname{wt}(x)=16$.

Although the condition given in Proposition 7 can be characterized by the vanishing of a coefficient in the weight enumerator of genus 3 (see [27]), we have not been able to prove Proposition 7 directly, without classifying all extremal doubly even self-dual codes of length 40.

In Table 6 , we list the numbers $N$ (dim) of extremal doubly even self-dual codes such that subcodes generated by codewords of weight 8 have dimension dim. The dimension is the same as the 2-rank of the 1-(40,8,57) design formed by the codewords of weight 8 . 
Table 6: Dimensions of subcodes generated by codewords of weight 8

\begin{tabular}{c|c|c|c|c}
\hline $\operatorname{dim}$ & 17 & 18 & 19 & 20 \\
\hline$N(\operatorname{dim})$ & 5 & 1 & 14 & 16450 \\
\hline
\end{tabular}

\section{$4 \quad$ Extremal self-dual codes of length 38}

Let $D$ be a doubly even self-dual code of length 40 . Let $C$ be the code obtained from $D$ for which some particular pair of coordinates $i, j$ are 00 and 11 and deleting these coordinates. Then $C$ is a self-dual code of length 38 . Here, we say that $C$ is obtained from $D$ by subtracting coordinates $i, j$. In addition, any self-dual code of length 38 is obtained from some doubly even self-dual code of length 40 by subtracting some two coordinates (see [9]). Due to the computational complexity, we only completed a classification of extremal self-dual codes of length 38. Note that there are at least 13644433 inequivalent self-dual codes of length 38 [11].

Any extremal self-dual code $C$ of length 38 and its shadow $S$ have one of the following weight enumerators [10]:

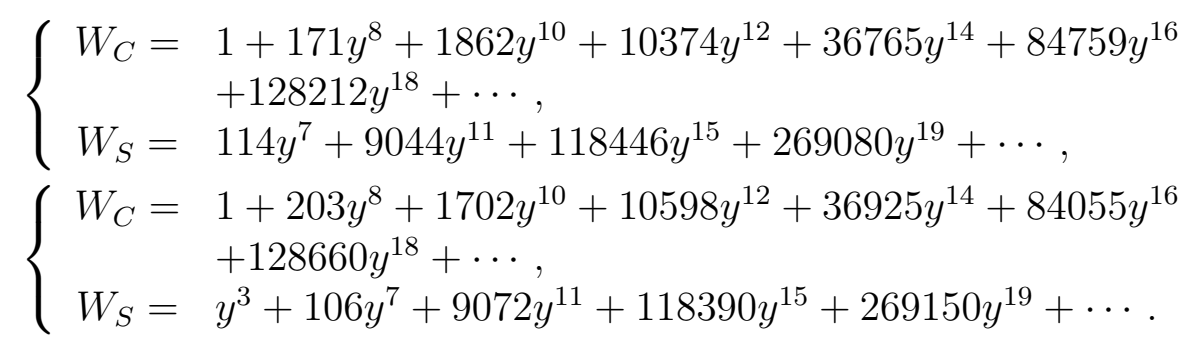

Although the following two lemmas are somewhat trivial, it is useful in finding extremal self-dual codes of length 38 .

Lemma 8. Any extremal self-dual code of length 38 with weight enumerator (7) (resp. (8)) is obtained from some extremal doubly even self-dual code of length 40 (resp. some doubly even self-dual code of length 40 containing one codeword of weight 4) by subtracting some two coordinates.

Proof. Let $C$ be an extremal self-dual code of length 38 with weight enumerator (7) (resp. (8)). By Proposition 2, a doubly even self-dual code $C^{*}$ of length 40 is constructed. In addition, by (7) (resp. (8)), $C^{*}$ is extremal (resp. $C^{*}$ contains one codeword of weight 4). The code $C$ is obtained from $C^{*}$ by subtracting the last two coordinates. The result follows.

For the remainder of this section, we suppose that $D$ is either an extremal doubly even self-dual code of length 40 or a doubly even self-dual code of length 40 containing one codeword of weight 4 . Also, let $D_{i, j}$ denote the self-dual code of length 38 obtained from $D$ by subtracting two coordinates $i, j$. 
Lemma 9. Let $M(D)$ be the matrix with rows composed of the codewords of weight 8 in $D$, where the $(1,0)$-matrix $M(D)$ is regarded as a matrix over $\mathbb{Z}$.

(1) Suppose that $D$ is extremal. Then the $(i, j)$-entry of $M(D)^{T} M(D)$ is zero if and only if $D_{i, j}$ is extremal.

(2) Suppose that $D$ contains one codeword $x$ of weight 4 . Then the $(i, j)$-entry of $M(D)^{T} M(D)$ is zero and the pair of coordinates $i, j$ in $x$ are 10 or 01 if and only if $D_{i, j}$ is extremal.

Proof. There is a codeword of weight 8 in $D$ for which the coordinates $i, j$ are 11 if and only if $D_{i, j}$ contains a codeword of weight 6 . Suppose that $D$ contains one codeword $x$ of weight 4 . The coordinates $i, j$ in $x$ are 11 (resp. 00) if and only if $D_{i, j}$ contains a codeword of weight 2 (resp. 4).

By Lemma 9, from all inequivalent extremal doubly even self-dual codes and all inequivalent doubly even self-dual codes containing one codeword of weight 4 , we constructed extremal self-dual codes of length 38 which need be checked further for equivalences. Then we checked equivalences among these codes using the method similar to that given in Section 2. Finally, we have the following:

Proposition 10. There are 2744 inequivalent extremal self-dual codes of length 38. Of these 1730 have weight enumerator (7) and 1014 have weight enumerator (8).

Remark 11. A classification of extremal self-dual codes of length 38 was very recently obtained in [1] by somewhat different techniques. This was indicated by Jon-Lark Kim in a private communication [17].

In Table 7, we list the numbers $N$ (\# Aut) of extremal self-dual codes with automorphism groups of order \# Aut for both weight enumerators (7) and (8).

Table 7: Number of extremal self-dual codes of length 38

\begin{tabular}{c|c|c|c|c}
\hline \multicolumn{5}{c}{ (\# Aut, $N(\#$ Aut) $)$} \\
\hline \multicolumn{5}{c}{ Weight enumerator $(7)$} \\
\hline$(1,1480)$ & $(4,30)$ & $(9,1)$ & $(24,4)$ & $(342,1)$ \\
$(2,177)$ & $(6,7)$ & $(12,5)$ & $(36,1)$ & \\
$(3,15)$ & $(8,7)$ & $(18,1)$ & $(168,1)$ & \\
\hline \multicolumn{5}{c}{ Weight enumerator $(8)$} \\
\hline$(1,773)$ & $(4,38)$ & $(12,3)$ & $(24,10)$ & $(216,1)$ \\
$(2,145)$ & $(6,10)$ & $(14,1)$ & $(144,1)$ & $(504,1)$ \\
$(3,21)$ & $(8,8)$ & $(21,1)$ & $(168,1)$ & \\
\hline
\end{tabular}

Some historical comments (July 27, 2012). A classification of extremal self-dual codes of length 38 was completed in [1], and a classification of all self-dual codes of length 38 was completed in [7]. The paper [1] was submitted before this paper was submitted, and the paper [7] was submitted after this paper was submitted. 


\section{Acknowledgements}

The authors would like to thank Jon-Lark Kim for providing information on [1].

\section{References}

[1] C. Aguilar-Melchor, P. Gaborit, J.-L. Kim, L. Sok and P. Solé. Classification of extremal and $s$-extremal binary self-dual codes of length 38. IEEE Trans. Inform. Theory, 58:2253-2262, 1983.

[2] E. F. Assmus, Jr. and V. Pless. On the covering radius of extremal self-dual codes. IEEE Trans. Inform. Theory, 29:359-363, 1983.

[3] R. T. Bilous. Enumeration of the binary self-dual codes of length 34. J. Combin. Math. Combin. Comput., 59:173-211, 2006.

[4] R. T. Bilous and G. H. J. van Rees. An enumeration of self-dual codes of length 32. Des. Codes, Cryptogr., 26:61-86, 2002.

[5] W. Bosma and J. Cannon. Handbook of Magma Functions. Department of Mathematics, University of Sydney, Available online at http://magma .maths. usyd.edu.au/magma/.

[6] S. Bouyuklieva. Some optimal self-orthogonal and self-dual codes. Disc. Math., 287:1-10, 2004.

[7] S. Bouyuklieva and I. Bouyukliev. An algorithm for classification of binary self-dual codes. IEEE Trans. Inform. Theory, 58:3933-3940, 1983.

[8] R. Brualdi and V. Pless. Weight enumerators of self-dual codes. IEEE Trans. Inform. Theory, 37:1222-1225, 1991.

[9] J. H. Conway, V. Pless and N. J. A. Sloane. The binary self-dual codes of length up to 32: a revised enumeration. J. Combin. Theory Ser. A, 60:183-195, 1992.

[10] J. H. Conway and N. J. A. Sloane. A new upper bound on the minimal distance of self-dual codes. IEEE Trans. Inform. Theory, 36:1319-1333, 1990.

[11] M. Harada and A. Munemasa. Classification of self-dual codes of length 36. Advances Math. Communications, 6:229-235, 2012.

[12] M. Harada and A. Munemasa. Database of Self-Dual Codes. Available online at http://www.math.is.tohoku.ac.jp/ munemasa/selfdualcodes.htm.

[13] M. Harada, A. Munemasa and K. Tanabe. Extremal self-dual [40, 20, 8] codes with covering radius 7. Finite Fields Appl., 10:183-197, 2004.

[14] M. Harada and M. Ozeki. Extremal self-dual codes with the smallest covering radius. Disc. Math., 215:271-281, 2000.

[15] W. C. Huffman. On the classification and enumeration of self-dual codes. Finite Fields Appl., 11:451-490, 2005.

[16] H. J. Kim. Self-dual codes with automorphism of order 3 having 8 cycles. Des. Codes Cryptogr., 57:329-346, 2010. 
[17] J.-L. Kim. private communication. April 25, 2011.

[18] O. D. King. The mass of extremal doubly-even self-dual codes of length 40. IEEE Trans. Inform. Theory, 47:2558-2560, 2001.

[19] F. J. MacWilliams, N. J. A. Sloane and J. G. Thompson. Good self dual codes exist. Disc. Math., 3:153-162, 1972.

[20] C. L. Mallows and N. J. A. Sloane. An upper bound for self-dual codes. Inform. Control, 22:188-200, 1973.

[21] R. L. Miller. Doubly Even Codes. Available online at http://www.rlmiller.org/de_codes/.

[22] M. Ozeki. Jacobi polynomials for singly even self-dual codes and the covering radius problems. IEEE Trans. Inform. Theory, 48:547-557, 2002.

[23] V. Pless. A classification of self-orthogonal codes over GF(2). Disc. Math., 3:209-246, 1972.

[24] V. Pless and N. J. A. Sloane. On the classification and enumeration of self-dual codes. J. Combin. Theory Ser. A, 18:313-335, 1975.

[25] E. M. Rains. Shadow bounds for self-dual codes. IEEE Trans. Inform. Theory, 44:134-139, 1998.

[26] E. Rains and N. J. A. Sloane. Self-dual codes. In Handbook of Coding Theory, V. S. Pless and W. C. Huffman (Editors), pages 177-294, Elsevier, Amsterdam, 1998.

[27] B. Runge. Codes and Siegel modular forms. Disc. Math., 148:175-204, 1996.

[28] V. Y. Yorgov. Binary self-dual codes with automorphisms of odd order. Problems Inform. Transmission, 19:260-270, 1984. translated from Problemy Peredachi Informatsii, 19:11-24, 1983 (Russian).

[29] V. Y. Yorgov and N. Zyapkov. Doubly even self-dual [40, 20, 8]-codes with an automorphism of odd order. Problems Inform. Transmission, 32:253-257, 1997. translated from Problemy Peredachi Informatsii, 32:41-46, 1996 (Russian). 